\title{
VHF antenna pattern characterization by the observation of meteor head echoes
}

\author{
Toralf Renkwitz, Carsten Schult, and Ralph Latteck \\ Leibniz-Institute of Atmospheric Physics, Schloss-Str. 6, 18225 Kuehlungsborn, Germany \\ Correspondence to: Toralf Renkwitz (renkwitz@iap-kborn.de)
}

Received: 28 September 2016 - Discussion started: 7 October 2016

Revised: 13 December 2016 - Accepted: 27 December 2016 - Published: 14 February 2017

\begin{abstract}
The Middle Atmosphere Alomar Radar System (MAARSY) with its active phased array antenna is designed and used for studies of phenomena in the mesosphere and lower atmosphere. The flexible beam forming and steering combined with a large aperture array allows for observations with a high temporal and angular resolution. For both the analysis of the radar data and the configuration of experiments, the actual radiation pattern needs to be known. For that purpose, various simulations as well as passive and active experiments have been conducted. Here, results of meteor head echo observations are presented, which allow us to derive detailed information of the actual radiation pattern for different beam-pointing positions and the current health status of the entire radar. For MAARSY, the described method offers robust beam pointing and width estimations for a minimum of a few days of observations.
\end{abstract}

\section{Introduction}

The Middle Atmosphere Alomar Radar System (MAARSY) was built in 2009/2010 by the Leibniz-Institute of Atmospheric Physics (IAP) on the northern Norwegian island Andøya $\left(69.3^{\circ} \mathrm{N}, 16.04^{\circ} \mathrm{E}\right)$ for improved studies of various atmospheric heights at high spatial and temporal resolution. The main target regions are the troposphere/lower stratosphere and the mesosphere/lower ionosphere, typically 1-25 and $50-120 \mathrm{~km}$, respectively.

MAARSY's main active phased antenna array consists of 433 yagi antennae (see Fig. 1), which are connected to their individual transceiver modules, allowing for independent phase and amplitude control. Such a configuration allows for both flexible pulse-to-pulse steering and forming of the radar beam by appropriately selecting amplitude and phase distribution over the array elements. A detailed description of the radar and its properties is given by Latteck et al. (2012), while recent geophysical investigations with MAARSY regarding layered phenomena in the mesosphere have been presented in Latteck and Strelnikova (2015) and Sommer et al. (2016).

The knowledge of the current radiation pattern characteristics is important for both the design of experiments, e.g. specific experimental settings, and is even more crucial for the analysis of radar data. The most important points to know are the beam-pointing accuracy, shape and width of the beam, the antenna gain and the position and intensity of side lobes.

For the validation of MAARSY's radiation pattern, various passive and active experiments were already conducted. In passive experiments, cosmic radio emissions from our galaxy, as well as distinct radio sources like radio galaxies, supernova remnants and the diffuse background, were observed. Subsequently, the derived intensity maps covering a northern declination from 10 to $90^{\circ}$ were compared to the detailed temperature Global Sky Model by de OliveiraCosta et al. (2008), using a reference map involving the simulated radiation pattern of MAARSY. The simulation of MAARSY's radiation pattern considering the mechanical structure, soil properties and antenna coupling were performed using the well-accepted Numerical Electromagnetics Code (NEC 4.1) for different arctic weather conditions. This enabled us to derive the pointing accuracy and beam width for different antenna array sizes (Renkwitz et al., 2012) and estimated the antenna array gain by knowing the absolute intensity flux of the observed radio sources (Renkwitz, 2014). Furthermore, observations of distinct radio sources like Cassiopeia A allow absolute phase calibration for the individual 


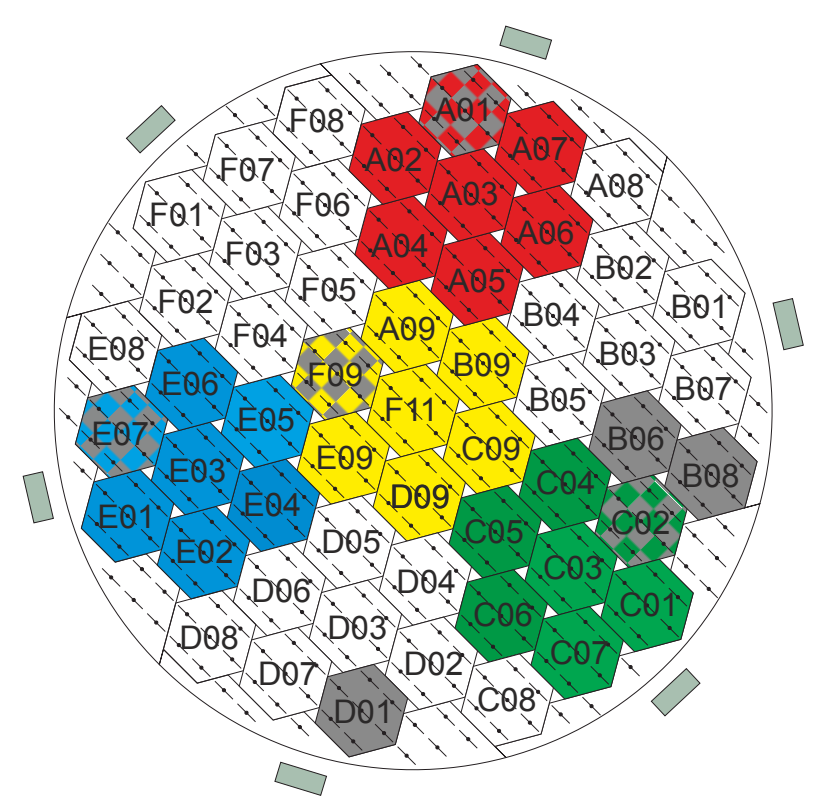

Figure 1. Sketch of MAARSY's main antenna array, consisting of 433 yagi antennae. The anemone subarray groups A, C, E and M, each composed of 49 antennae, are individually colour coded, while 7 antennae form a hexagon, e.g. D-01. Besides the total antenna array, the subarray groups marked in grey are used for reception in the examined experiment mst006.

subgroups of an antenna array as described for MAARSY in Chau et al. (2014). Knowledge of the individual antenna array subgroup phases is essential for integrating them into synthetic arrays, providing different beam shapes and widths as well as the interferometric analysis of the data, e.g. to locate the mean angle of arrival of the radar echo.

Additionally, active experiments were conducted in which the Earth's moon and large artificial satellites were used as radar targets, as described in Renkwitz et al. (2013), as well as scattering off a sounding rocket's payload (Renkwitz et al., 2015) more recently. In general, these experiments have shown a good agreement with the simulated radiation pattern, though some discrepancies have been found in the estimated beam widths and negligible inaccuracy of the beam pointing. The cause for the slightly increased beam width was assumed to be a matter of the existing mixed polarization during the system upgrade in 2012/2013, converting from linear to circular polarization. This widening of the radiation pattern was also indicated in thorough NEC simulations.

In this paper we present the methodology that has been used to augment our knowledge of the actual threedimensional radiation pattern of the MAARSY radar by analysing meteor head echo observations. Here, the main aim is to present a robust approach to estimate and validate the simulated radiation pattern using the angularly resolved statistical distribution of meteor head echo trajectories.
For specular meteor radars, the angular distribution of detected trails allows us to map the general coverage of the used radars. This coverage distribution can be seen in widebeam meteor radars using, e.g. one single beam (Stober et al., 2011), multiple beams like the SAAMER/DRAAMER installations (Fritts et al., 2010, 2012), but also in narrow-beam antenna arrays like the MU radar (Nakamura et al., 1991). The observation of specular meteors, however, does not allow the actual beam shape to be derived, but gives an indication of the maximum radial coverage off the nominal pointing direction. This is caused by the necessity of having perpendicularity between the emitted radio wave and the meteor trajectory. With this, for specular meteors only one single point of the plasma trail perpendicular to the radar beam is seen for the duration of the event.

Thus, only a subset of all existing meteors are seen by a single monostatic specular meteor radar. Furthermore, most of the specular meteors are typically seen around $50^{\circ}$ elevation angle and below due to the increasing observation volume for the meteor ablation heights. Especially, for the zenith-near directions, the geometry for suitable meteor trajectories perpendicular to the radio wave is very limited. Thus, the angular statistical appearance of specular meteors cannot be used for the beam pattern validation.

However, the perpendicularity requirement does not hold for the observation of meteor head echoes when the plasma around the meteor head acts as an isotropic scatterer. This allows echoes for every single pulse to be emitted by the radar towards the meteor head on its trajectory through the dense ionosphere, namely the $\mathrm{E}$ and $\mathrm{D}$ regions. Therefore, meteor head echoes can be used to map the radiation pattern by the angular distribution of detected echoes for a certain amount of time. Meteor head echoes, however, are severely weaker than specular meteor echoes scattering off the meteor trail and therefore high-power large-aperture radars are needed to compensate for the lower efficiency of this scattering process. Meteor head observations are described for various radar systems of sufficient power-aperture products, e.g. in Pellinen-Wannberg and Wannberg (1994), Janches et al. (2000), Chau and Woodman (2004), Pellinen-Wannberg (2005), Chau et al. (2009), Kero et al. (2011) and Schult et al. (2013).

In the following, section meteor head echo observations with MAARSY are briefly described, followed by the methodology of this experiment using meteor head echo observations for radiation pattern validation. The proposed method is shown for two case studies in which MAARSY was operational with the entire antenna array and for a short period when about $20 \%$ of the array was non-functional resulting in a distorted radiation pattern. The results underline the exceptional value of this analysis to monitor the health status of the radar as a byproduct of routine observations. Finally, a discussion and conclusions are given. 


\section{Meteor head echo observations with MAARSY}

The MAARSY radar is one of the few radar systems that are capable of observing meteor head echoes as has been successfully demonstrated during the Geminids meteor shower in 2010 (Schult et al., 2013). Contrary to other radars that observed meteor head echoes on a campaign basis, MAARSY recently completed its second year of nearly continuous observations. For example, this extraordinarily rich meteor data set allows for the generation of radiant maps and velocity distributions but, even more interestingly, for the estimation of dynamical meteor masses seen by the radar and thus its potential contribution to an improved meteor input function, which is presented in detail in Schult et al. (2016). The advantage of such meteor head echo measurements by radars is their uniqueness, as they do not depend on weather conditions or the time of day like optical instruments. For MAARSY, meteor head echo observations are performed with either specialized or multi-purpose experiments. The most interesting details of the multi-purpose experiment used during the examined period are shown in Table 1. Within this experiment, the troposphere and mesosphere regions were monitored together. The echo intensity is typically derived for the entire antenna array, while the radial and angular position of the meteor head echo trajectories are derived by interferometric means using smaller subarrays. For transmission, the entire antenna array was operated at maximum output power without employing an amplitude taper. For reception, various subarrays, hexagons and anemones comprising of 7 and 49 antennas respectively have been used for the interferometric analysis. The benefit of using different sizes of subarrays and the baseline between them is connected to their individual gain and beam width as well as the resulting interferometric unambiguous angular range. The use of closely spaced smaller subarrays (B-06, B-08, C-02; see array sketch in Fig. 1), each with a $30^{\circ}$ beam width, facilitates an unambiguous angular range of approximately $15.6^{\circ}$. In addition, widely spaced anemone subarrays (A, C, E, M), each with an $11^{\circ}$ beam width, provide more gain in the main beam direction. Thus, the closely spaced hexagons permit the rough angular location of the event, while using the anemones allows for the detection of weaker events, as well as precise phase and thus position information of the observed target with their longer baseline lengths.

In Fig. 2 is an extraordinary long lasting and intense example of meteor head echoes observed with MAARSY. In the left panel is the signal-to-noise ratio, while in the right panel the angularly resolved trajectory of this meteor event with colour-coded altitude is depicted. Additionally, the first and second null and side lobes of the MAARSY two-way radiation pattern are marked with black and red circles. This specific meteor event was seen from roughly $105 \mathrm{~km}$ down to $82 \mathrm{~km}$ altitude, traversing MAARSY's first side lobe. In contrast to the meteor head echo trajectory, a single arbitrary
Table 1. Details of the monitoring experiment mst006 also used to observe meteor head echoes, accompanied by the list of used subarray groups (see Fig. 1 for comparison). The antennae Y1, Y2, Y3 and ALW64 are external antennae and are not used in this study.

\begin{tabular}{ll}
\hline Parameter & Value \\
\hline $\begin{array}{l}\text { Power-aperture product } \\
\text { Pulse repetition }\end{array}$ & $9.7 \mathrm{MWm}{ }^{2}$ \\
Frequency & $1000 \mathrm{~Hz}$ \\
Range resolution & $450 \mathrm{~m}$ \\
Sampling range & $\approx 7-148 \mathrm{~km}$ \\
Beam directions & $\phi=0^{\circ}, \theta=0^{\circ} ;$ \\
& $\phi=185^{\circ}, \theta=12.4^{\circ}$ \\
Pulse code & $16-$ bit complementary \\
Coherent integration & 2 \\
Nyquist frequency & $15.6 \mathrm{~Hz}$ \\
Subarray groups & $\mathrm{A}, \mathrm{Y} 1, \mathrm{C}, \mathrm{Y} 2, \mathrm{E}, \mathrm{Y3}, \mathrm{M}$, \\
& $\mathrm{A}-01, \mathrm{~B}-06, \mathrm{C}-02, \mathrm{D}-01 \mathrm{E}-07$, \\
& $\mathrm{ALW64}, \mathrm{B}-08$ \\
\hline
\end{tabular}

point was added, marked by a red asterisk, which would be seen for a specular meteor event.

Due to the high velocity of the observed targets the received signals need to be decoded Doppler-corrected for the individual complementary codes to ensure proper reconstruction of the signals and thus the individual meteor trajectory (see e.g. Kero et al., 2012; Schult et al., 2016, and references within).

The detected power of meteor head echoes for the same radar parameters vary significantly from one event to the other due to the meteor's orientation and entry velocity to the Earth atmosphere, its size and composition, which therefore requires normalization of the individual data. First attempts to use normalized meteor head echo intensities for some events observed with MAARSY were compared to simulated radiation pattern cross sections as described in Chau et al. (2014). Here, quite good agreement for the main beam and the first side lobe were found, both in shape and width. However, as previously quoted, this approach was limited to the piecewise comparison of a few individual meteor trajectories.

\section{Methodology and experiment}

The initial premise for this study was to automatically analyse the trajectory data without additional discrimination or selection of individual meteor head echo events and their trajectories. The detected intensities, or better the sufficient signal-to-noise ratio, is a necessary requirement for the calculation of the trajectory, but is not used in the subsequent analysis described here. Contrary to the previously mentioned method of inferring the two-dimensional radiation pattern from the detected intensities of selected meteor trajectories, here the statistical occurrence of trajectory 

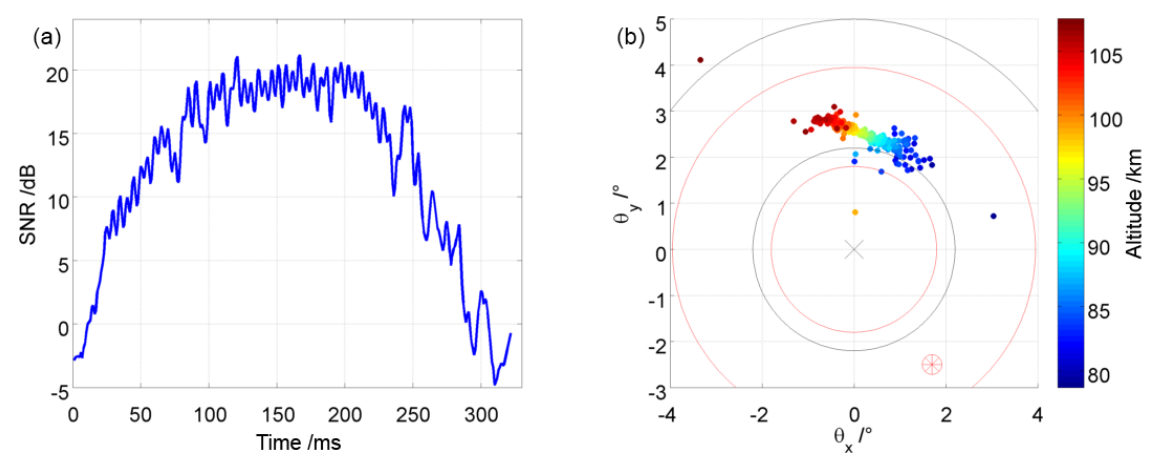

Figure 2. Example of a long lasting and intense meteor head echo observed with MAARSY on 18 January 2016 at 12:46 UT. (a) Detected signal-to-noise ratio, (b) trajectory of the meteor in colour-coded altitude for zenith angles $\theta_{x}$ and $\theta_{y}$. Black and red circles indicate the position of the first and second null and side lobes of the radiation pattern. The red asterisk mark an arbitrary position of a specular meteor event.

points per defined angular bin is used to deduce the threedimensional radiation pattern.

The assumption is that, as long as the radar parameters, especially beam pointing and width, are not changed significantly, the radiation pattern should be reflected in the angular occurrence of the events. As previously noted, the detectability of meteor head echoes depends on the volume illuminated by the radar and the reception depends on the selection of subarray groups (beam width, gain), but also on the available baselines to recover the unambiguous position of the meteors. Thus, the angular occurrence, or count rate, of the meteor head echo positions within the given unambiguous angular range is only determined by the actual radiation pattern.

The data we use in this study were collected with the MAARSY radar between 1 December 2013 and 26 February 2014 with the monitoring experiment mst006 (see Table 1). With this experiment radar measurements in two distinct beam directions were performed, pointing towards zenith and off-vertical towards $\phi=185^{\circ}, \theta=12.4^{\circ}$.

For the reliable reconstruction of a meteor's trajectory a minimum of 11 points are defined, but may consist of a few hundred points for larger, but rare, examples as previously shown in Fig. 2. During the entire period, the average length of derived trajectories accounts for 28 points.

For this experiment, the occurrence of all derived meteor trajectory points are calculated for angular bins of a resolution of $0.2 \times 0.2^{\circ}$ resolution and ablation heights of 85 to $115 \mathrm{~km}$ are used. The increasing volume for greater zenith angle off-sets is negligible for zenith near directions, $<0.1 \mathrm{~dB}$ for $5^{\circ}$, but more crucial for $\theta=15^{\circ}$ accounting for $-0.3 \mathrm{~dB}$. The resulting occurrence map is smoothed (9point-median) and interpolated where necessary to derive the complete three-dimensional intensity and shape of the main beam and the first side lobes as well as the beam width.

During the period studied here, on 31 December one of the six containers housing the radar hardware for the anemone group $\mathrm{F}$ and two hexagon groups of the centre anemone $\mathrm{M}$ were not operational for either reception or transmission, but operation was restored on 7 January. This period of restricted operability is individually investigated to underline the reliability and sensitivity of this method.

\subsection{Angular occurrence of meteor head echo trajectories for a period of 3 months}

For the nearly 3 months of full functionality in total, 141118 meteor head echo trajectories, accounting for more than $4 \times 10^{6}$ trajectory points for the zenith beam and the southwards tilted beam, were derived and used to generate angular occurrence maps.

The calculated occurrence of meteor trajectories for the zenith pointing radar beam is depicted in Fig. 3. The occurrence map is accompanied by contour lines of the simulated two-way radiation pattern. While the entire antenna array, superposed of 433 nominal antennae, are used for transmission, the latter 49 antenna subgroups are the largest directly sampled subarrays of MAARSY and are used on reception for the final interferometric examination of the trajectory estimation. A very similar distribution was derived for the tilted radar beam, pointing to $\phi=185^{\circ}, \theta=12.4^{\circ}$, but it is not shown separately as no additional information is apparent.

As for MAARSY, every array element has its own transceiver module that sensitively reacts to the adjacent antennae and their emissions. For example, in the case of unfavourably superposed mutual coupling of the antennae resulting in excessive reflected power, the monitoring circuitry temporarily disables modules during the experiments. Additionally, in some cases amplifier modules may get damaged over time, but are repaired during the next maintenance. The health status of MAARSY's transmitter modules is stored in a database, which for the period analysed in this paper unfortunately got lost due to a hard disk failure. Therefore, we performed thorough simulations in which nearly $5 \%$ of the array elements were disabled (quasi-randomly distributed). These simulations should better match MAARSY's health status during that period and actually also predict a better 


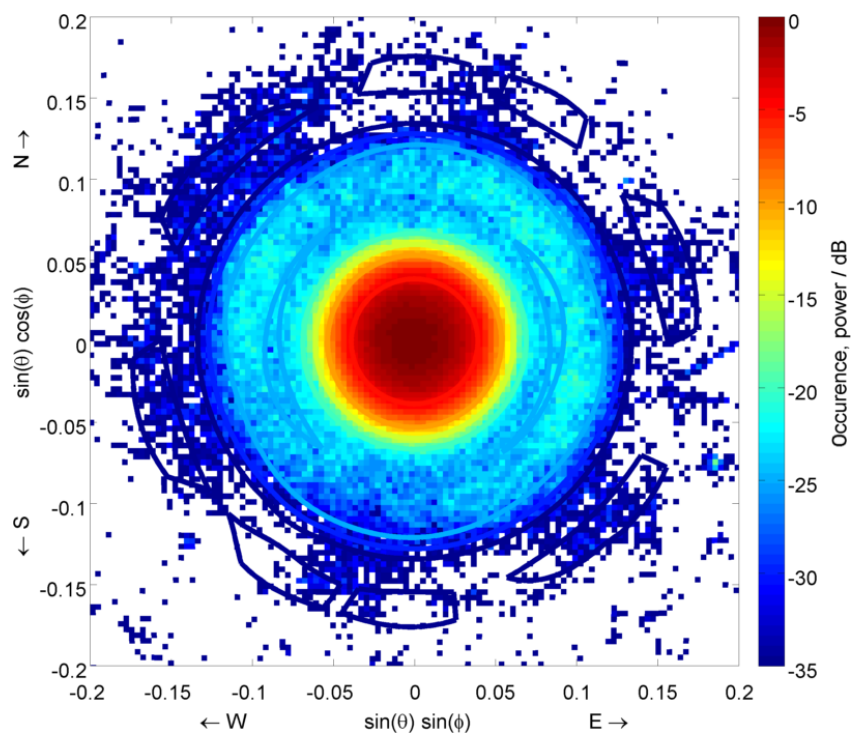

Figure 3. Angular occurrence rate of meteor head echo trajectory points for zenith beam pointing observed with MAARSY during a period of almost 3 months and overlaid by the simulated radiation pattern.

agreement with the observations. Initial simulations incorporating all 433 antennae have shown a lower side lobe attenuation of about $3 \mathrm{~dB}$.

For both cases, the occurrences agree well to the simulated radiation pattern for the main lobe and first side lobe, which is highlighted in Fig. 4. There, the cross sections in the north-south and east-west planes for both beam directions are shown as well as the simulated pattern reference.

The observed beam shape and width for both pointing directions match the simulations, while a slight broadening is seen for the tilted beam, which is caused by a decrease in the effective antenna area. The broadening factor can be estimated by the cosine of the off-boresight pointing, which agrees to the observed broadening of $0.1^{\circ}$. Besides this, a slight beam-pointing error of approximately $0.1^{\circ}$ towards the south-west is visible in the figure.

A precise beam-pointing estimation was achieved by calculating the median of all original trajectory points, before sorting the data into $0.2^{\circ}$ bins. The beam-pointing positions for the entire period of proper operation were calculated to $\phi=246.7^{\circ}, \theta=0.12^{\circ}$ and $\phi=185.6^{\circ}, \theta=12.47^{\circ}$ for the zenith pointing and tilted beam. The offset of the nominal beam pointing may consistently be accounted for by setting both beam directions to $0.12^{\circ}$ south-west of the initial direction.

\subsection{Estimated radiation pattern for a period of a few days}

Contrary to the previous section, in which the MAARSY radar was in normal operation nominally using the entire an-

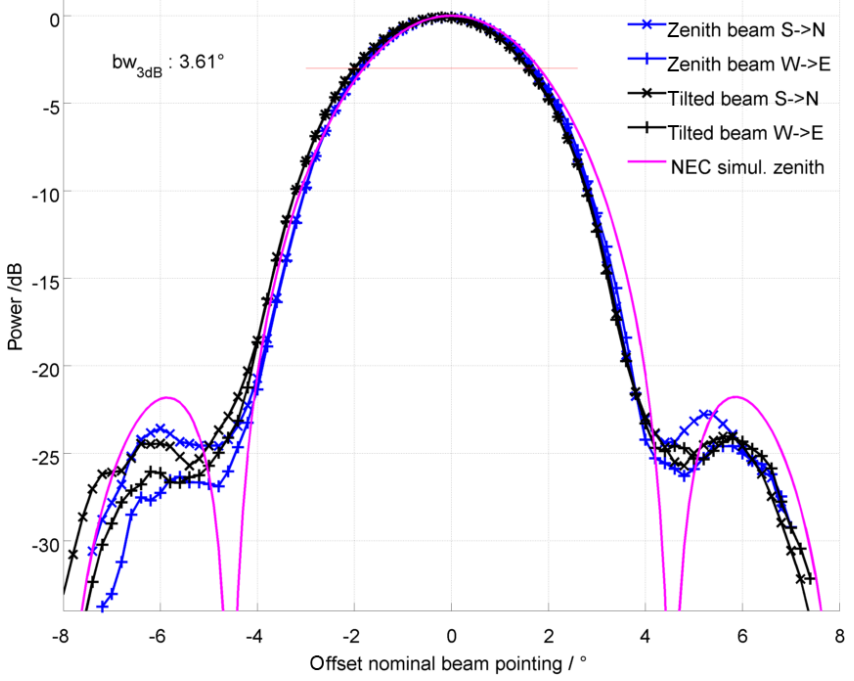

Figure 4. Cross sections through the angular occurrence rate for both beam-pointing directions, $\phi=0^{\circ}, \theta=0^{\circ}$ (blue) and $\phi=185^{\circ}$, $\theta=12.4^{\circ}$ (black) with respect to their nominal position, compared to the simulated radiation pattern (magenta).

tenna array, here the period from 1 to late 7 January 2014 is investigated. During that period, container F failed and therefore $20 \%$ of the antenna array was not available (subarray groups F-01 to F-11; see Fig. 1). This inoperability affected the transmission and reception, in addition to the illumination of the target volume and loss of the hexagon F-09, which is also sampled for potential interferometric use. The latter does not affect the recovery of the meteor trajectories as the explicit hexagon is not part of the smallest essential baseline group (B-06, B-08, C-02).

For this period of 1 week, approximately 250000 reliable trajectory points for each beam-pointing direction were derived and analysed. The occurrence map for this period for the tilted beam is shown in Fig. 5, while the vertical beam is the same. As in the earlier figures, the occurrence maps have been superimposed by the simulated radiation pattern. For a horizontal cut through the main beam, i.e. contour line (yellow) of $-13 \mathrm{~dB}$, the normally circular shape of the main beam is clearly deformed to an oval shape due to the large proportion of inactive antennae. The longer axis of the oval is oriented along the direction of the missing array elements, north-west-south-east, which agrees with the inverse relationship of beam width and maximum extent of incorporated array elements. For both beam directions, the occurrence maps appear to be similar in terms of shape and spread around the nominal beam-pointing direction. This underlines the proper function of the remaining radar hardware and antenna array in use and allows for the detailed analysis shown in Fig. 6. In the left panel the south-north cross section is shown for both beam-pointing directions with respect to their nominal beam-pointing position, while in the right panel the south-west to north-east cross section is depicted. The latter 


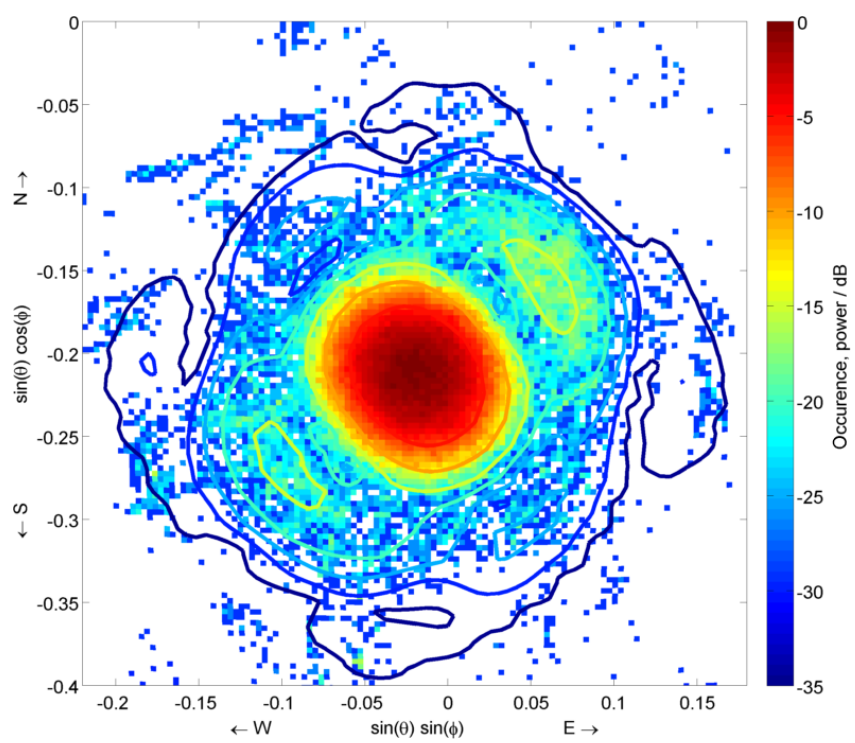

Figure 5. Angular occurrence rate for the tilted beam $\left(\phi=185^{\circ}\right.$, $\theta=12.4^{\circ}$ ) overlaid by the simulated radiation pattern for January 2014 when about $20 \%$ of the antenna array was non-functional.

is especially interesting as the prominent side lobes are generated in these directions, seen in both the observations and the simulations. For both beam-pointing directions the simulated pattern proposes less side lobe suppression than we actually see in this analysis, especially for the south-westnorth-east cross section, though they still agree fairly well for the south-north cross section. It is worth noting that earlier observations of scattering off a sounding rocket's payload (Renkwitz et al., 2015), however, indicated a better agreement of side lobes' intensity to the simulations. The position of the first null, especially for the SW-NE cross section, seems to be slightly shifted outwards.

To complement the earlier findings and to underline the sensitivity of the described method, the occurrences of meteor trajectory points for 15 December 2013 for both beampointing directions $\phi=0^{\circ}, \theta=0^{\circ}$ and $\phi=185^{\circ}, \theta=12.4^{\circ}$ are depicted in Fig. 7. On that day, about 2850 meteor head echo trajectories, consisting of 82000 points, were derived in total for both beam directions. The high number of detected trajectories and visible distinct spurs next to the main beam positions are related to the day of these measurements around the maximum of the Geminids meteor shower. The detected distribution already matches the Gaussian-like shape of the radiation pattern fairly well, but for the precise derivation of parameters like beam width a larger data set should be used. With the assumption of pattern symmetry and referencing to the centre of the beam, it would be possible to average the occurrences in azimuth to overcome the low count rates. However, the median beam-pointing direction for this single day is estimated to be about $0.17^{\circ}$ south-westwards off the nom-
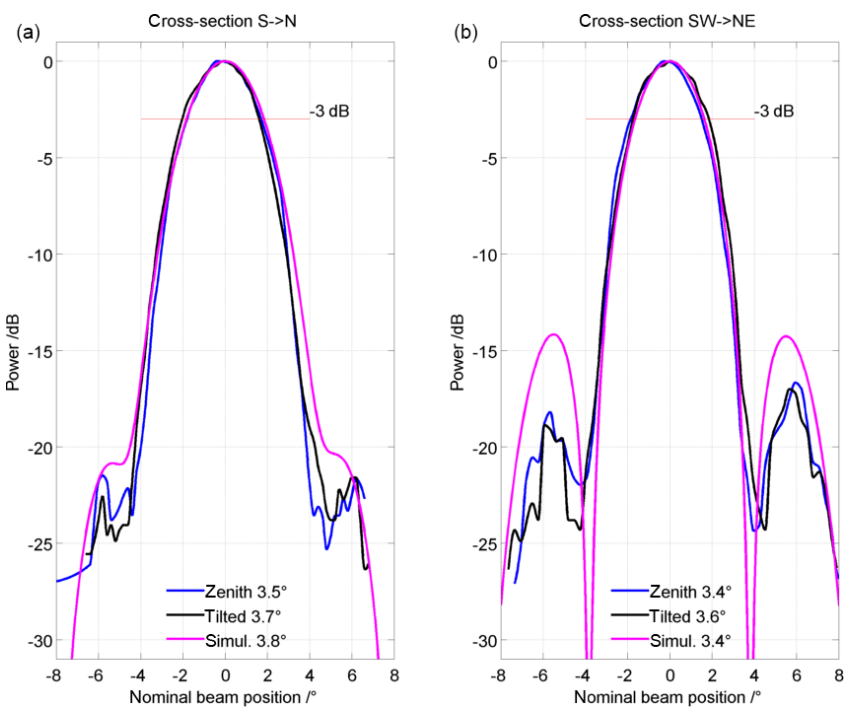

Figure 6. Cross sections through the angular occurrence rate for both beam directions compared to the simulated radiation pattern for the period of 1-8 January 2014. The simulated and estimated beam widths are shown at the bottom.

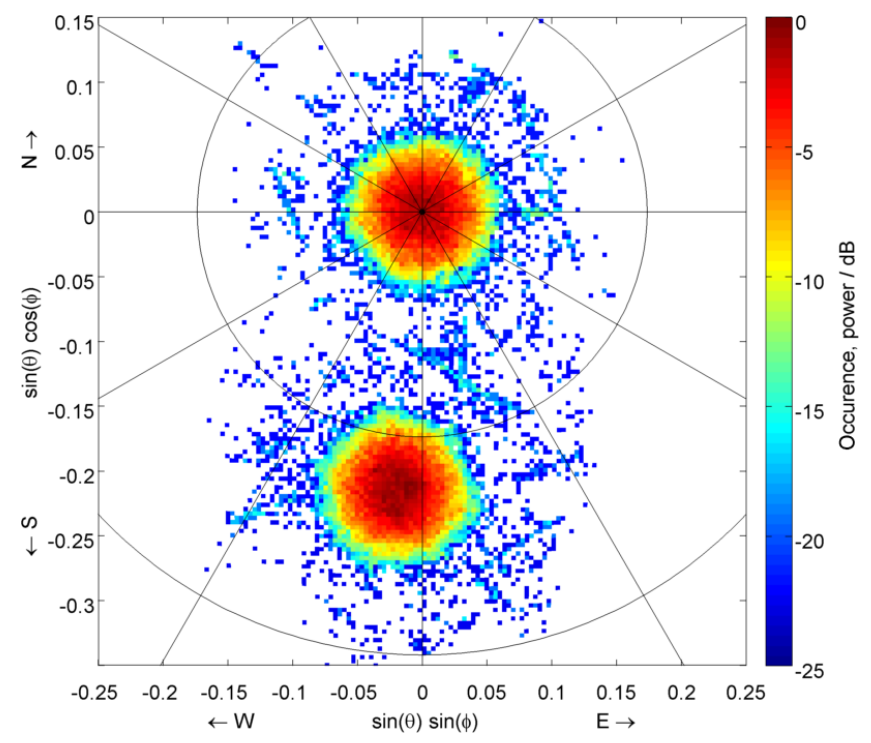

Figure 7. Angular occurrence of meteor head echo trajectory points observed on 15 December 2013 for two beam-pointing directions, $\phi=0^{\circ}, \theta=0^{\circ}$ and $\phi=185^{\circ}, \theta=12.4^{\circ}$.

inal direction, which is in good agreement with the value estimated for the entire period of nearly 3 months.

\section{Discussion}

The described method seems to be suitable for deriving the actual radiation pattern by analysing the occurrence of meteor head echo trajectory points. For the rough estimation of the pattern, we have shown occurrences for just a single day. 
Although it occurred during the maximum of the Geminids shower, it proved the general reliability of the method. Of course, it is certainly recommended to use as much data as is available to improve the statistics for both the reconstruction of all individual trajectories and the parameters derived from the total occurrences.

Altogether, the estimated beam widths for the individual periods correspond well to the simulation, though a minor broadening can be distinguished. Broadening can be caused by amplitude tapering (typically used to gain additional side lobe suppression, which was not consciously applied, as well as missing active antennae at the rim of the antenna array) or improper phase distribution. Besides the beam width, other remarkable points in the radiation pattern are the position and attenuation of nulls. The first null next to the main lobe, at about $4.3^{\circ}$, is clearly seen in the simulations, but is not as pronounced in the observations for the initially presented period of nominal functionality. A potential cause of this inferior behaviour is assumed to be connected to random phase and amplitude variations over the antenna array. However, the position of the first null for the south-west-north-east cross section is well accentuated for the shown period when container F failed and thus $20 \%$ of the transceiver modules were inoperable. As previously noted, at times transceiver modules and their antennae randomly distributed over the antenna array are temporarily or permanently disabled. As the database of transceiver modules' health status was lost, we assumed a quasi-random distribution of nearly $5 \%$ nonfunctional array elements in the simulations. These missing array elements modify the ideal radiation pattern, but are repaired during the next regular maintenance of the radar. With these assumed missing elements, the detected side lobe intensities agree better than with the radiation pattern of the entire array of nominal functionality. For the period in which about $20 \%$ of the antenna array were inactive, higher discrepancies are seen in the south-west-north-east cross section, perpendicular to the orientation of reduced array diameter. Furthermore, the detected and simulated beam widths differ slightly during that time, which is likely caused by the comparably small data set and uncertainties in the radar's health and thus in the simulations.

In Renkwitz (2014), the influence of malfunctioning transceiver modules, and thus the temporary loss of antenna array elements, was examined in addition to random amplitude and phase errors of all array elements. For the loss of 21 randomly distributed antenna modules a beam broadening of up to $0.2^{\circ}$ was seen, while the position of the side lobes were mostly unchanged and only their absolute amplitudes varied. For random amplitude and phase errors $( \pm 1 \mathrm{~dB}$ and $\pm 10^{\circ}$ ) a negligible beam broadening was seen, but the intensity and positions of side lobes were significantly modified. Therefore, the estimated minor discrepancies seem to be well in line with the previously mentioned study.

With the unambiguous interferometric angular range of maximum $15.6^{\circ}$ around the nominal beam position in this ex- periment, the main lobe and first three side lobes of transmit radiation pattern should be covered. A plausible flaw might origin from intense meteor head echoes originating from $18^{\circ}$ off the main beam, corresponding to the third radiation pattern side lobe. Such meteor head echoes are likely misplaced within the unambiguous angular range, impairing the derived occurrence maps. However, with the reasonably wellshaped form of the main and side lobe derived from the occurrence rates, the meteor positions generally seem to be recovered reliably, which is connected to correct decoding and unwrapping of observed phases between the individual small subarrays used in the interferometric analysis. A systematically flawed determination of the meteor trajectories would directly result in an anomalous shape of the contour maps. However, minor phase errors and temporary variations result in slightly wrong localization of the echoes, which likely also spoil the nulls in the radiation pattern.

Still, the most likely cause of the seen overestimated side lobe attenuation is probably related to the initial detection of meteor events, which is performed on the data of the entire antenna array. Meteor events off the nominal beam direction are already less frequently seen within the entire antenna array than with a smaller subarray like the anemones. The reasoning for the initial detection of meteor events using the entire array is related to the high likelihood of also detecting the same event in all subarrays. Complementing the initial meteor detection by also searching in the anemone and hexagon data might provide a higher number of meteor events off the nominal beam position.

The beam-pointing accuracy was consistently estimated to be in the order of $0.12^{\circ}$ south-westwards off the nominal beam direction for all examined data. A potential cause might be a slightly flawed phase calibration of the individual subarrays or the calibration of the radar containers housing the equipment of the individual subarrays. However, the effect of this error seems to be negligible as the nominal halfpower beam width is almost 40 times larger.

Other radar experiments facilitating an equivalent quality to verify the radiation pattern depend on either distinct scattering structures of known properties, which are rare for the wavelength used here, or highly sensitive receive-only observations of cosmic radio emissions and the ability to perform angular scans. With this, the generation of meteor head echo occurrence maps as a byproduct within standard monitoring experiments seems to be very adjuvant, provides valuable information about the health status of the radar and validates the radiation pattern.

\section{Conclusions}

In this paper, we presented analysis results for two periods of meteor head echo observations. The continuous operation of MAARSY with a multi-functional monitoring experiment also allows meteor head echoes to be extensively observed. 
The observation of meteor head echoes with MAARSY is motivated by performing the first continuous meteor head echo measurements, e.g. to derive the climatology, radiant maps, dynamical meteor masses and to investigate meteor showers in detail. As a byproduct of this rich data set, the deduction of the radiation pattern was envisaged.

Earlier methods related the detected intensities of individual meteor head echo trajectories in piecewise comparisons to cross sections of the simulated radiation pattern. Contrary, the method described here relies only on the derived positions of the meteor head echo trajectories, but not on the detected intensities. The angular distribution of meteor head trajectories and count rates for $0.2 \times 0.2^{\circ}$ bins are determined and occurrence maps are generated. These occurrence maps are assumed to be highly equivalent to the combined twoway radiation pattern.

For the cases shown in this study, a zenith beam and a $12.4^{\circ}$ tilted beam were used during normal operation of the entire array and a period when about $20 \%$ of the transceiver modules failed, which mainly influenced the transmission pattern. The occurrences during these periods and the individual beams were compared to the simulated radiation pattern, which revealed a remarkable good agreement. Especially, for the 1-week period of restricted functionality, the derived occurrence map matches exceptionally well to the simulations with its deformation of the main lobe and emerging side lobes. A minor deficiency seems to be existent in the derived absolute intensity of the side lobes, which is most likely related to the involved meteor detection method basing on the measurements with the entire antenna array.

Nevertheless, the obvious performance of this method makes it to a very sensitive and reliable tool to monitor the radar system's health as a byproduct, which was underlined by the occurrence map derived for just one day.

Acknowledgements. The authors explicitly acknowledge the contribution of Jorge L. Chau for adjuvant discussions and suggestions related to the interferometric analysis in general and to meteor head echo observations as well as Svenja Sommer who was involved in deriving absolute calibration phases for MAARSY. Furthermore, we express our gratitude to the Andøya Space Center for their permanent support for the operation and maintenance of the MAARSY radar. The radar development was supported by the German grant 01 LP 0802A of Bundesministerium für Bildung und Forschung, while the meteor head echo observations are supported by the grant STO 1053/1-1 of the Deutsche Forschungsgemeinschaft (DFG). We also like to thank the referees for giving valuable comments to improve this paper.

Edited by: M. Rapp

Reviewed by: two anonymous referees

\section{References}

Chau, J. L. and Woodman, R. F.: Observations of meteor-head echoes using the Jicamarca $50 \mathrm{MHz}$ radar in interferometer mode, Atmos. Chem. Phys., 4, 511-521, doi:10.5194/acp-4-5112004, 2004.

Chau, J. L., Galindo, F. R., Heinselman, C. J., and Nicolls, M. J.: Meteor-head echo observations using an antenna compression approach with the $450 \mathrm{MHz}$ Poker Flat Incoherent Scatter Radar, J. Atmos. Sol.-Terr. Phy., 71, 636-643, doi:10.1016/j.jastp.2008.08.007, 2009.

Chau, J. L., Renkwitz, T., Stober, G., and Latteck, R.: MAARSY multiple receiver phase calibration using radio sources, J. Atmos. Sol.-Terr. Phy., 118, 55-63, doi:10.1016/j.jastp.2013.04.004, 2014.

de Oliveira-Costa, A. Tegmark,, M., Gaensler, B. M., Jonas, J., Landecker, T. L., and Reich, P.: A model of diffuse galactic radio emission from $10 \mathrm{MHz}$ to $100 \mathrm{GHz}$, Mon. Not. R. Astron. Soc., 338, 247-260, doi:10.1111/j.1365-2966.2008.13376.x, 2008.

Fritts, D. C., Janches, D., Iimura, H., Hocking, W. K., Mitchell, N. J., Stockwell, R. G., Fuller, B., Vandepeer, B., Hormaechea, J., Brunini, C., and Levato, H.: Southern Argentina Agile Meteor Radar: System design and initial measurements of largescale winds and tides, J. Geophys. Res.-Atmos., 115, 112-130, doi:10.1029/2010JD013850, 2010.

Fritts, D. C., Janches, D., Iimura, H., Hocking, W. K., Bageston, J. V., and Pene, N. M.: Drake Antarctic Agile Meteor Radar first results: Configuration and comparison of mean and tidal wind and gravity wave momentum flux measurements with Southern Argentina Agile Meteor Radar, J. Geophys. Res.-Atmos., 117, 105-121, doi:10.1029/2011JD016651, 2012.

Janches, D., Mathews, J. D., Meisel, D. D., and Zhou, Q. H.: Micrometeor observations using the Arecibo $430 \mathrm{MHz}$ radar, I. Determination of ballistic parameter from measured Doppler velocity and deceleration results, Icarus, 145, 53-63, doi:10.1006/icar.1999.6330, 2000.

Kero, J., Szasz, C., Nakamura, T., Meisel, D. D., Ueda, M., Fujiwara, Y., Terasawa, T., Miyamoto, H., and Nishimura, K.: First results from the 2009-2010 MU radar head echo observation programme for sporadic and shower meteors: the Orionids 2009, Mon. Not. R. Astron. Soc., 416, 2550-2559, doi:10.1111/j.13652966.2011.19146.x, 2011.

Kero, J., Szasz, C., Nakamura, T., Terasawa, T., Miyamoto, H., and Nishimura, K.: A meteor head echo analysis algorithm for the lower VHF band, Ann. Geophys., 30, 639-659, doi:10.5194/angeo-30-639-2012, 2012.

Latteck, R. and Strelnikova, I.: Extended observations of polar mesosphere winter echoes over Andøya $\left(69^{\circ} \mathrm{N}\right)$ using MAARSY, J. Geophys. Res.-Atmos., 120, 8216-8226, doi:10.1002/2015JD023291, 2015.

Latteck, R., Singer, W., Rapp, M., Vandepeer, B., Renkwitz, T., Zecha, M., and Stober, G.: The new MST radar on Andøya: System description and first results, Radio Science, 47, doi:10.1029/2011RS004775, 2012.

Nakamura, T., Tsuda, T., Tsutsumi, M., Kita, K., Uehara, T., Kato, S., and Fukao, S.: Meteor wind observations with the MU radar, Radio Science, 26, 857-869, doi:10.1029/91RS01164, 1991.

Pellinen-Wannberg, A.: Meteor head echoes - observations and models, Ann. Geophys., 23, 201-205, doi:10.5194/angeo-23201-2005, 2005. 
Pellinen-Wannberg, A. and Wannberg, G. Meteor observations with the European Incoherent Scatter UHF Radar, J. Geophys. Res., 99, 11379-11390, doi:10.1029/94JA00274, 1994.

Renkwitz, T.: Evaluation and validation of a novel MST-Radar for studying atmospheric 3D structures, PhD thesis, University of Rostock, Rostock, Germany, 2014.

Renkwitz, T., Singer, W., Latteck, R., Stober, G., and Rapp, M.: Validation of the radiation pattern of the Middle Atmosphere Alomar Radar System (MAARSY), Adv. Radio Sci., 10, 245-253, doi:10.5194/ars-10-245-2012, 2012.

Renkwitz, T., Stober, G., Latteck, R., Singer, W., and Rapp, M.: New experiments to validate the radiation pattern of the Middle Atmosphere Alomar Radar System (MAARSY), Adv. Radio Sci., 11, 283-289, doi:10.5194/ars-11-283-2013, 2013.

Renkwitz, T., Schult, C., Latteck, R., and Stober, G.: Validation of the radiation pattern of the VHF MST radar MAARSY by scattering off a sounding rocket's payload, Adv. Radio Sci., 13, 4148, doi:10.5194/ars-13-41-2015, 2015.
Schult, C., Stober, G., Chau, J. L., and Latteck, R.: Determination of meteor-head echo trajectories using the interferometric capabilities of MAARSY, Ann. Geophys., 31, 1843-1851, doi:10.5194/angeo-31-1843-2013, 2013.

Schult, C., Stober, G., Chau, J. L., and Janches, D.: Results of the first continuous meteor head echo survey at polar latitudes, Icarus, in review, 2016.

Sommer, S., Stober, G., and Chau, J. L.: On the angular dependence and scattering model of polar mesospheric summer echoes at VHF, J. Geophys. Res.-Atmos., 121, 278-288, doi:10.1002/2015JD023518, 2016.

Stober, G., Singer, W., and Jacobi, C.: Cosmic radio noise observations using a mid-latitude meteor radar, J. Atmos. Sol.-Terr. Phy., 73, 1069-1076, doi:10.1016/j.jastp.2010.07.018, 2011. 